\title{
Programa de treinamento vocal para locutores de rádio
}

\author{
Voice training program for radio presenters
}

\author{
Soraya Mahmoud Farghaly' ${ }^{1}$, Claudia Regina Furquim de Andrade ${ }^{2}$
}

\begin{abstract}
RESUMO
Objetivo: Propor e avaliar a eficácia de um Programa Fonoaudiológico para Formação de Locutores de Rádio, aplicado aos alunos de um curso profissionalizante de radialista. Métodos: Foram participantes 70 adultos subdivididos em dois grupos, pareados quanto ao sexo e idade: Grupo I (grupo de pesquisa), constituído por 35 adultos, alunos do curso de locução; Grupo II (grupo controle) composto por 35 adultos, que espontaneamente se disponibilizaram a participar da pesquisa. O Programa foi estruturado em sete módulos com duração de sete aulas semanais. Esse Programa foi fundamentado na estimulação de 11 aspectos, os quais foram trabalhados seqüencialmente. Foram realizadas três análises, sendo: análise objetiva da voz, por meio da extração da frequiência fundamental, análise perceptivo-auditiva do uso vocal e análise objetiva da velocidade de fala na leitura. Resultados: Os resultados indicaram que os grupos diferenciaram-se quanto ao uso vocal e à velocidade de fala na leitura. Em relação à análise do uso vocal, os grupos diferenciaram-se para os parâmetros de qualidade vocal, loudness, ressonância, coordenação pneumofonoarticulatória, articulação, modulação e ritmo de leitura. Quanto à análise da velocidade de fala, os grupos diferenciaram-se tanto para o número de palavras/ minuto como de sílabas/minuto. Em relação à análise objetiva da voz, para o grupo de pesquisa houve diferenças entre o pré e pósaplicação do programa, com redução da freqüência fundamental. Conclusão: Os resultados indicam que o Programa foi eficaz na sua proposta e os parâmetros que apresentaram melhoras são aqueles específicos para a boa locução.
\end{abstract}

Descritores: Treinamento da voz; Voz; Rádio; Fala; Acústica da fala; Percepção da fala; Métodos; Desenvolvimento de programas

\section{INTRODUÇÃO}

Embora o referencial teórico da literatura nacional e internacional sobre a atuação fonoaudiológica com a voz profissional seja amplo, o enfoque do trabalho fonoaudiológico com os locutores de rádio é extremamente reduzido. Essa área tem recebido pouca atenção quanto à estruturação metodológica, seja em relação aos procedimentos utilizados, ou à avaliação dos resultados. A escassez de estudos com a presença de grupo controle, a falta de padronização dos procedimentos e a imprecisão metodológica no tocante a incompatibilidade na definição e na seleção das técnicas utilizadas, é bastante evidente nos trabalhos dessa área.

Apesar de não existir um protocolo padronizado para medir os resultados funcionais do trabalho fonoaudiológico com os locutores de rádio, existe um consenso entre os poucos pesquisadores do assunto, sobre a importância do trabalho de

Trabalho realizado na Universidade de São Paulo - USP - São Paulo (SP), Brasil.

(1) Mestre, Professora do Curso de Rádio e TV da Academia Paulista Anchieta da Universidade Bandeirante - UNIBAN - São Paulo (SP), Brasil.

(2) Professora Titular do Departamento de Fisioterapia, Fonoaudiologia e Terapia Ocupacional da Faculdade de Medicina da Universidade de São Paulo - USP - São Paulo (SP), Brasil.

Endereço para correspondência: Claudia Regina Furquim de Andrade. R. Cipotânea, 51, Cidade Universitária, São Paulo - SP, CEP 05360-160.

E-mail: clauan@usp.br

Recebido em: 26/11/2007; Aceito em: 5/9/2008 voz e promoção da saúde vocal ${ }^{(1-7)}$.

O Serviço Nacional de Aprendizagem Comercial (SENAC) oferece em sua grade curricular o curso de Qualificação Profissional de Radialista - Setor Locução. Esse curso é de caráter intensivo, exclusivamente profissionalizante e autorizado pelo Conselho Nacional de Educação a fornecer o Registro Profissional junto à Delegacia Regional do Trabalho.

$\mathrm{O}$ trabalho fonoaudiológico com os alunos desse curso incentivou a presente pesquisa no sentido de estruturar metodologicamente uma proposta de trabalho e avaliar cientificamente os resultados deste trabalho.

$\mathrm{O}$ uso adequado dos diferentes registros vocais está intimamente relacionado com a saúde vocal do profissional da voz $^{(8-9)}$. Os profissionais da voz devem adaptar seu padrão vocal em função da demanda de comunicação para uma qualidade vocal adequada e saudável, e minimizar os fatores de risco para alterações vocais ${ }^{(10-13)}$.

O padrão diferenciado da fala do locutor é promovido via emprego de marcas vocais específicas, com o objetivo de atrair o ouvinte para o que está sendo comunicado ${ }^{(14)}$.

O locutor adapta seu padrão vocal, caracterizado por um contorno prosódico característico e elementos enfáticos, de acordo com as diferentes situações de comunicação, ao qual é exposto ${ }^{(15-16)}$. Esse profissional necessita buscar recursos que o levem ao aprimoramento da sua locução, reestruturando constantemente a sua fala ${ }^{(17)}$.

A redução da freqüência fundamental com pitch mais grave 
é bastante utilizada entre os locutores, como forma de caracterizar o discurso e obter melhor resultado nas transmissões das mensagens. Em relação à eficácia do treinamento vocal, diversos estudos enfatizam a necessidade de um treinamento de voz bem estruturado e elaborado para futuros profissionais da $v^{(18-25)}$.

A atividade de leitura pós-treinamento vocal pode ser considerada como resultado de uma elaboração possibilitando uma melhor caracterização vocal ${ }^{(26-27)}$.

A velocidade de fala é um importante indicativo do grau de fluência, definida como o fluxo contínuo e suave da produção da fala ${ }^{(28)}$, medida pelo fluxo de palavras por minuto e o fluxo de sílabas por minuto ${ }^{(29)}$. Uma emissão fluente corresponde a um alto grau de continuidade, entendida como a quantidade de correspondência entre intenção e emissão. Estudos na área indicam o fluxo da velocidade de fala em atividade de fala espontânea, tanto para palavras/min, como em sílabas $/ \mathrm{min}^{(30)}$.

Considerando a relevância dessa revisão de literatura, assim como a escassez metodológica em propostas de cursos de treinamento vocal para os locutores de rádio, foi delineada a presente pesquisa, com o objetivo de propor e avaliar a eficácia de um Programa Fonoaudiológico para Formação de Locutores de Rádio (PFFLR), direcionado aos alunos de um curso de locução. Esta pesquisa busca fundamentar cientificamente uma proposta de curso para locutores de rádio, na área de treinamento e capacitação vocal, e contribuir para a objetividade e redução do ensaísmo nas práticas fonoaudiológicas.

\section{MÉTODOS}

\section{Participantes}

Foram considerados participantes desta pesquisa 70 adultos, com idades entre 18 e 45 anos, ensino fundamental concluído, sem distinção de sexo, subdivididos em dois grupos. O grupo de pesquisa (GI) foi constituído por 35 adultos (19 do sexo masculino e 16 do sexo feminino), todos eles alunos do curso profissionalizante de locução do SENAC; e o grupo controle (GII), constituído por 35 adultos (19 do sexo masculino e 16 do sexo feminino) que espontaneamente se dispuseram em participar da pesquisa, não freqüentadores de nenhum curso profissionalizante de locução. Os grupos foram pareados quanto ao sexo e idade.

O critério de inclusão dos participantes de ambos os grupos foi apresentar triagem negativa para alteração da comunicação. Era necessário que todos participantes não reportassem déficits associados aos quadros de distúrbios da comunicação, que não fossem nem exercessem atividades como locutores profissionais ou amadores.

\section{Material}

Para a realização desta pesquisa, foram utilizados três protocolos elaborados com base nas literaturas específicas e adaptados pela autora deste estudo ${ }^{(4-6)}$, e um protocolo utilizado integralmente sem adaptações ${ }^{(28)}$.

Os protocolos utilizados foram: protocolo de triagem fonoaudiológica ${ }^{(4-6)}$ (Anexo 1), protocolo de avaliação objetiva da $\operatorname{voz}^{(4-6)}$ (Anexo 2), protocolo de avaliação do uso vocal ${ }^{(4-6)}$ (Anexo 3) e o protocolo para avaliação da fluência da fala ${ }^{(28)}$ (Anexo 4).

Foram utilizados o equipamento de gravação digitalizado de voz, o programa computadorizado de espectrografia acústica GRAM 5.7, os protocolos de avaliação ${ }^{(4-6,28)}$ e o Programa Fonoaudiológico para Formação de Locutores de Rádio (PFFLR).

As amostras de voz e de fala, obtidas a partir da aplicação dos protocolos específicos, foram gravadas digitalmente, por meio de um gravador digital Sony ${ }^{\circledR}$ (Portable MiniDisc Recorder MZ R 70) e um microfone profissional, electrocondensador, unidirecional, sensitividade $-40+2 \mathrm{~dB}$, SK Pró Áudio.

\section{Procedimento}

Os procedimentos de pesquisa só foram iniciados após os processos éticos pertinentes: assinatura do Termo de Consentimento Pós-Informação e o parecer com aprovação da Comissão de Ética para Análise de Projetos de Pesquisa do Hospital das Clínicas da Faculdade de Medicina da Universidade de São Paulo (CAPPesq $n^{\circ}$ 571/02). Todos os participantes envolvidos consentiram na realização dessa pesquisa e divulgação de seus resultados, conforme a Resolução 196/96.

Foram realizadas sessões individuais de avaliação, respectivamente dois dias antes (pré-teste) e dois dias depois (pósteste) da aplicação do Programa Fonoaudiológico para Formação de Locutores de Rádio (PFFLR). Na sessão individual de pré-teste, foi aplicado o protocolo de triagem fonoaudiológica e, em seguida, foram coletadas as amostras de voz e de fala, para aplicação dos protocolos específicos de voz e fala ${ }^{(4-6,28)}$. Na sessão individual de pós-teste, foram realizadas novas coletas para aplicação dos mesmos protocolos específicos.

O procedimento de coleta dos dados apresentou as seguintes etapas:

\section{Triagem fonoaudiológica}

Composta por sete provas diferenciadas: prova de Articulação; Linguagem; Voz; Fluência; Variações Fonoaudiológicas; Sistema Miofuncional e Audição, conforme protocolo adaptado de triagem fonoaudiológica ${ }^{(4-6)}$.

\section{Avaliação do perfil de voz e de fala}

Durante a coleta das amostras, os participantes permaneceram sentados, obedecendo à postura mais comum ao locutor radialista. O material de voz foi analisado sob o ponto de vista acústico e perceptivo-auditivo.

Para a avaliação objetiva da voz foi utilizado o programa espectrográfico GRAM 5.7, para a extração da freqüência fundamental por intermédio da emissão da vogal sustentada /a/, conforme o protocolo adaptado de avaliação objetiva da $\operatorname{voz}^{(4-6)}$.

A avaliação do uso vocal foi realizada a partir da leitura de dois pequenos textos no estilo notícia e comercial. O uso vocal foi avaliado dentro dos parâmetros de qualidade vocal, ataque vocal, pitch, loudness, ressonância, coordenação pneumo-fono-articulatória, articulação, modulação e ritmo 
de leitura, conforme o protocolo adaptado de avaliação do uso vocal ${ }^{(4-6)}$.

A avaliação da velocidade de fala em leitura foi realizada a partir da leitura de dois pequenos textos no estilo notícia e comercial, por meio do cálculo do fluxo de palavras/minuto e sílabas/minuto, em amostra composta por 200 sílabas fluentes, conforme protocolo para avaliação da fluência da fala ${ }^{(28)}$.

\section{Programa Fonoaudiológico para Formação de Locutores de Rádio (PFFLR)}

Este Programa é estruturado metodologicamente com duração e conteúdos pré-determinados, ministrado por meio de módulos fechados com objetivos direcionados e controlado em sua eficácia.

O PFFLR foi ministrado pela própria autora do estudo e fundamentado na estimulação de 11 aspectos, trabalhados seqüencialmente: propriocepção da voz; saúde vocal; ajustes vocais específicos; respiração; articulação; pontuação, inflexão vocal, ênfase, modulação, ritmo de leitura e imagem vocal.

O PFFLR foi aplicado ao grupo de pesquisa (GI) e composto por sete aulas semanais, estruturadas em sete módulos. A aplicação do PFFLR foi contínua e diária, os participantes foram orientados a realizar, no mínimo, uma vez por dia, os exercícios específicos de cada módulo. Os módulos foram evolutivos, independentemente do aproveitamento isolado em cada um deles.

Este programa foi elaborado na forma de suporte teórico, material áudio visual e ilustrativo e vivências. O limite máximo de faltas permitidas durante a aplicação do PFFLR foi de duas faltas, garantindo $75 \%$ de presença. Essa medida permitiu maior controle da eficácia do PFFLR, uma vez que três faltas implicariam a não realização de $25 \%$ do programa. Nos casos onde foi excedido esse limite, houve a exclusão do participante como sujeito da pesquisa, embora permanecesse como aluno frequientando as aulas.

Para o grupo controle (GII), os procedimentos de pré e pósteste foram idênticos ao grupo de pesquisa (GI), mantendo-se as mesmas condições de gravação. Foram realizadas duas sessões direcionadas, sendo uma de pré-teste e uma de pós-teste, onde foram coletadas as amostras de voz e de fala e aplicados os protocolos específicos ${ }^{(4-6,28)}$. O intervalo de tempo correspondente entre o pré e pós-teste foi de sete semanas, intervalo de tempo correspondente à aplicação do PFFLR.

As análises foram divididas em variáveis paramétricas e não paramétricas. Para a análise objetiva da voz e análise objetiva da velocidade de fala em leitura, foi utilizado o Teste t de Student para Dados Pareados e para a análise do uso vocal foi utilizado o Teste de Mann-Whitney, sendo adotado o nível de significância $p \leq 0,05$ para todas as análises.

\section{RESULTADOS}

Os resultados indicam que os grupos diferenciaram-se significativamente quanto ao uso vocal e a velocidade de fala na leitura.

A Tabela 1 descreve a média de distribuição dos valores e a caracterização do uso vocal na comparação inter-grupos no pré e pós-teste. Pode-se verificar que, em relação ao uso vocal, os grupos diferenciaram-se significativamente para os parâmetros de qualidade vocal, loudness, ressonância, coordenação pneumofonoarticulatória, articulação, modulação e ritmo de leitura, com adequação desses parâmetros para a locução, após a aplicação do Programa Fonoaudiológico para Formação de Locutores de Rádio (PFFLR).

Tabela 1. Caracterização inter-grupos quanto a média de distribuição e caracterização do uso vocal no pré e pós-teste

\begin{tabular}{|c|c|c|c|c|}
\hline Parâmetro & Grupo & $\mathrm{N}$ & Média & Significância \\
\hline \multirow{2}{*}{$\begin{array}{l}\text { Qualidade vocal } \\
\text { Pré-teste }\end{array}$} & Grupo I & 35 & 2,57 & \multirow[t]{2}{*}{$p=0,533$} \\
\hline & Grupo II & 35 & 2,69 & \\
\hline \multirow{2}{*}{$\begin{array}{l}\text { Qualidade vocal } \\
\text { Pós-teste }\end{array}$} & Grupo I & 35 & 3,0 & \multirow[t]{2}{*}{$p=0,001^{*}$} \\
\hline & Grupo II & 35 & 2,69 & \\
\hline \multirow{2}{*}{$\begin{array}{l}\text { Ataque vocal } \\
\text { Pré-teste }\end{array}$} & Grupo I & 35 & 2,83 & \multirow[t]{2}{*}{$p=0,645$} \\
\hline & Grupo II & 35 & 2,89 & \\
\hline \multirow{2}{*}{$\begin{array}{l}\text { Ataque vocal } \\
\text { Pós-teste }\end{array}$} & Grupo I & 35 & 3,0 & \multirow[t]{2}{*}{$\mathrm{p}=0,154$} \\
\hline & Grupo II & 35 & 2,89 & \\
\hline \multirow{2}{*}{$\begin{array}{l}\text { Pitch } \\
\text { Pré-teste }\end{array}$} & Grupo I & 35 & 2,71 & \multirow[t]{2}{*}{$p=0,235$} \\
\hline & Grupo II & 35 & 2,89 & \\
\hline \multirow{2}{*}{$\begin{array}{l}\text { Pitch } \\
\text { Pós-teste }\end{array}$} & Grupo I & 35 & 3,0 & \multirow[t]{2}{*}{$p=0,154$} \\
\hline & Grupo II & 35 & 2,89 & \\
\hline \multirow{2}{*}{$\begin{array}{l}\text { Loudness } \\
\text { Pré-teste }\end{array}$} & Grupo I & 35 & 2,57 & \multirow[t]{2}{*}{$p=0,694$} \\
\hline & Grupo II & 35 & 2,6 & \\
\hline \multirow{2}{*}{$\begin{array}{l}\text { Loudness } \\
\text { Pós-teste }\end{array}$} & Grupo I & 35 & 3,0 & \multirow[t]{2}{*}{$p=0,006^{*}$} \\
\hline & Grupo II & 35 & 2,6 & \\
\hline \multirow{2}{*}{$\begin{array}{l}\text { Ressonância } \\
\text { Pré-teste }\end{array}$} & Grupo I & 35 & 1,69 & \multirow[t]{2}{*}{$p=0,015^{\star}$} \\
\hline & Grupo II & 35 & 2,2 & \\
\hline \multirow{2}{*}{$\begin{array}{l}\text { Ressonância } \\
\text { Pós-teste }\end{array}$} & Grupo I & 35 & 2,91 & \multirow[t]{2}{*}{$p<0,001^{*}$} \\
\hline & Grupo II & 35 & 2,2 & \\
\hline \multirow{2}{*}{$\begin{array}{l}\text { Coordenação } \\
\text { pneumo-fono- } \\
\text { articulatória } \\
\text { Pré-teste }\end{array}$} & Grupo I & 35 & 1,23 & \multirow[t]{2}{*}{$p=0,127$} \\
\hline & Grupo II & 35 & 1,51 & \\
\hline \multirow{2}{*}{$\begin{array}{l}\text { Coordenação } \\
\text { pneumo-fono- } \\
\text { articulatória } \\
\text { Pós-teste }\end{array}$} & Grupo I & 35 & 2,83 & \multirow[t]{2}{*}{$p<0,001^{*}$} \\
\hline & Grupo II & 35 & 1,51 & \\
\hline \multirow{2}{*}{$\begin{array}{l}\text { Articulação } \\
\text { Pré-teste }\end{array}$} & Grupo I & 35 & 2,6 & \multirow[t]{2}{*}{$p=0,572$} \\
\hline & Grupo II & 35 & 2,49 & \\
\hline \multirow{2}{*}{$\begin{array}{l}\text { Articulação } \\
\text { Pós-teste }\end{array}$} & Grupo I & 35 & 3,0 & \multirow[t]{2}{*}{$p=0,001^{*}$} \\
\hline & Grupo II & 35 & 2,49 & \\
\hline Modulação & Grupo I & 35 & 1,34 & $p=0,136$ \\
\hline Pré-teste & Grupo II & 35 & 1,11 & \\
\hline Modulação & Grupo I & 35 & 2,77 & $p<0,001^{*}$ \\
\hline Pós-teste & Grupo II & 35 & 1,11 & \\
\hline Ritmo de leitura & Grupo I & 35 & 1,4 & $p=0,760$ \\
\hline & Grupo II & 35 & 1,34 & \\
\hline Ritmo de leitura & Grupo I & 35 & 2,89 & $p<0,001^{*}$ \\
\hline Pós-teste & Grupo II & 35 & 1,34 & \\
\hline
\end{tabular}

Teste estatístico utilizado: Teste de Mann-Whitney 
Tabela 2. Caracterização intrer-grupos quanto à média de distribuição da velocidade de fala em leitura de notícia e comercial no pré e pósteste

\begin{tabular}{|c|c|c|c|c|c|}
\hline Parâmetro & Grupo & $\mathrm{N}$ & Média & Desvio-Padrão & Significância \\
\hline \multirow{2}{*}{$\begin{array}{l}\text { Leitura de notícia-Fluxo de palavras/ } \\
\text { minuto } \\
\text { Pré-teste }\end{array}$} & Grupo I & 35 & 155,42 & 14,65 & \multirow[t]{2}{*}{$p=0,285$} \\
\hline & Grupo II & 35 & 151,14 & 18,39 & \\
\hline \multirow{2}{*}{$\begin{array}{l}\text { Leitura de notícia-Fluxo de palavras/ } \\
\text { minuto } \\
\text { Pós-teste }\end{array}$} & Grupo I & 35 & 164,32 & 12,0 & \multirow[t]{2}{*}{$p<0,001^{*}$} \\
\hline & Grupo II & 35 & 150,47 & 17,74 & \\
\hline \multirow{2}{*}{$\begin{array}{l}\text { Leitura de notícia-Fluxo de sílabas/ } \\
\text { minuto } \\
\text { Pré-teste }\end{array}$} & Grupo I & 35 & 343,0 & 32,24 & \multirow[t]{2}{*}{$p=0,289$} \\
\hline & Grupo II & 35 & 333,70 & 40,13 & \\
\hline \multirow{2}{*}{$\begin{array}{l}\text { Leitura de notícia-Fluxo de sílabas/ } \\
\text { minuto } \\
\text { Pós-teste }\end{array}$} & Grupo I & 35 & 361,36 & 26,12 & \multirow[t]{2}{*}{$p<0,001^{*}$} \\
\hline & Grupo II & 35 & 331,60 & 38,67 & \\
\hline \multirow{2}{*}{$\begin{array}{l}\text { Leitura de comercial-Fluxo de } \\
\text { palavras/minuto } \\
\text { Pré-teste }\end{array}$} & Grupo I & 35 & 136,46 & 12,22 & \multirow[t]{2}{*}{$p=0,254$} \\
\hline & Grupo II & 35 & 132,34 & 17,29 & \\
\hline \multirow{2}{*}{$\begin{array}{l}\text { Leitura de comercial-Fluxo de } \\
\text { palavras/minuto } \\
\text { Pós-teste }\end{array}$} & Grupo I & 35 & 141,19 & 10,46 & \multirow[t]{2}{*}{$p=0,009^{*}$} \\
\hline & Grupo II & 35 & 131,54 & 18,26 & \\
\hline \multirow{2}{*}{$\begin{array}{l}\text { Leitura de comercial-Fluxo de sílabas/ } \\
\text { minuto } \\
\text { Pré-teste }\end{array}$} & Grupo I & 35 & 323,69 & 28,67 & \multirow[t]{2}{*}{$p=0,248$} \\
\hline & Grupo II & 35 & 313,78 & 41,38 & \\
\hline \multirow{2}{*}{$\begin{array}{l}\text { Leitura de comercial-Fluxo de sílabas/ } \\
\text { minuto } \\
\text { Pós-teste }\end{array}$} & Grupo I & 35 & 336,21 & 24,73 & \multirow[t]{2}{*}{$p=0,003^{*}$} \\
\hline & Grupo II & 35 & 309,66 & 43,97 & \\
\hline
\end{tabular}

Teste estatístico utilizado: Teste t de Student para dados pareados

Tabela 3. Caracterização intra-grupo (Gl) quanto à média de distribuição da freqüência fundamental da voz $\left(\mathrm{F}_{0}\right)$ no pré e pós-teste

\begin{tabular}{lcccc}
\hline Parâmetro & Média & $\mathrm{N}$ & $\begin{array}{c}\text { Desvio- } \\
\text { Padrão }\end{array}$ & Significância \\
\hline $\mathrm{F}_{0}$ Pré-teste & 144,23 & 35 & 43,73 & $\mathrm{p}=0,041^{*}$ \\
$\mathrm{~F}_{0}$ Pós-teste & 138,86 & 35 & 43,21 & \\
\hline
\end{tabular}

Teste estatístico utilizado: Teste t de Student para dados pareados

A Tabela 2 apresenta a média de distribuição dos valores da velocidade de fala em leitura na comparação inter-grupos no pré e pós-teste. Os resultados indicam que os grupos diferenciaram-se significativamente, tanto para o número de palavras/minuto como de sílabas/minuto, com aumento na velocidade de fala, nas duas formas de textos trabalhados (notícia e comercial).

A Tabela 3 apresenta a média de distribuição dos valores da freqüência fundamental $\left(\mathrm{F}_{\mathrm{o}}\right)$ na comparação intra-grupo (GI), de acordo com os valores de pré e pós-teste. Observa-se que GI apresentou diferenças estatisticamente significantes entre o pré e pós-aplicação do PFFLR, com redução da frequiência fundamental.

\section{DISCUSSÃO}

Com relação ao agravamento da voz para a locução como forma de caracterizar o discurso, conforme observado na presente pesquisa pós-aplicação do Programa Fonoaudiológico para Formação de Locutores de Rádio (PFFLR), há concordâncias com diversos apontamentos da literatura ${ }^{(18-22,26-27)}$.
A avaliação do uso vocal indica que o PFFLR foi eficaz para sete dos nove parâmetros avaliados. Esta avaliação permite saber como o locutor pode utilizar os recursos vocais da melhor forma possível, contribuindo para a saúde vocal ${ }^{(4-7)}$.

Dentre os parâmetros que indicaram significância, a ressonância aparece com eficácia para $94,28 \%$ dos participantes, por meio da qual, o locutor consegue mais nuanças em sua locução, contemplando a questão estética da voz ${ }^{(14-17)}$.

A adequação do parâmetro de coordenação pneumofonoarticulatória obtida com eficácia para 91,43\% dos participantes, também é apontada na literatura ${ }^{(14-15,17)}$.

A atuação do locutor implica uma fala inteligível e precisa, sem distorções de sons ou padrão articulatório mal-definido. A presente pesquisa indica adequação de $100 \%$ para o parâmetro de articulação, compatível com os achados de outros estudos $^{(4-7)}$.

A modulação é um dos marcadores vocais mais importantes na locução, fazendo toda a diferença para manter interesse no que está sendo dito, atingindo o ouvinte de forma mais efetiva. Em relação a esse parâmetro, os resultados indicaram que o PFFLR foi eficaz para $88,57 \%$ dos participantes. Diversos estudos também evidenciam que o locutor, como um profissional da voz, apresenta um contorno entoacional característico, com grande número de elementos enfáticos ${ }^{(14-15,17)}$.

A avaliação do uso vocal realizada nesta pesquisa evidenciou que o locutor apresenta um marcador vocal que é treinado, por meio de recursos vocais específicos. Os ajustes vocais utilizados na locução são resultantes de uma elaboração vocal, que traduz a marca individual do locutor.

A pesquisa concorda com a literatura em relação aos di- 
versos estudos sobre a importância do treinamento vocal para profissionais da voz ${ }^{(18-22,26-27)}$.

Existem na literatura pouquíssimos trabalhos sobre a medida objetiva da velocidade de fala em atividade de leitura, com uma extrema escassez quando se referem aos locutores de rádio. Observa-se na literatura que muitos trabalhos desenvolvidos com a velocidade de fala, da forma que está sendo aqui avaliada, são da área da fluência.

Os resultados apresentados nesta pesquisa evidenciaram que a média de distribuição da velocidade de fala em leitura de notícia foi de 164,32 palavras/min e 361,36 sílabas/min, e em leitura de comercial foi de 141,19 palavras/min e 336,21 sílabas/min. Esses resultados quando comparados com estudos da área ${ }^{(30)}$ indicam que a velocidade de fala para leitura é maior do que a velocidade da fala espontânea.

\section{CONCLUSÃO}

A aplicação do Programa Fonoaudiológico para Formação de Locutores de Rádio (PFFLR), por meio da avaliação objetiva da voz, do uso vocal e da velocidade de fala na leitura, permitiu concluir que o programa foi eficaz na sua proposta para as avaliações do uso vocal e da velocidade de fala na leitura, uma vez que esses parâmetros diferenciaram os grupos de forma significante.

O PFFLR também permitiu concluir que em uma proposta de curso para formação de locutores, a frequiência fundamental da voz deve ter sua abordagem melhor investigada, porém não descartada, uma vez que esse parâmetro diferenciou de forma significante o grupo de pesquisa na comparação pré e pós-aplicação do PFFLR. Há falta de parâmetros que garanta que intervalo de variação deva ser considerado significativo para a redução da frequiência fundamental, após aplicação de técnicas específicas.

Esse estudo também permitiu observar que a atuação fonoaudiológica com os locutores de rádio deve ser estruturada com base nos parâmetros que indicaram significância relevante: qualidade vocal, loudness, ressonância, coordenação pneumofonoarticulatória, articulação, modulação, ritmo de leitura e velocidade de fala na leitura.

Quanto ao parâmetro velocidade de fala, há falta de valores de referência da velocidade de fala em atividade de leitura para adultos, de uma maneira geral, e específicos, para a atividade do locutor. Com base nos resultados obtidos no presente estudo, uma proposta de curso para formação de locutores deve contemplar técnicas específicas para a adequação da velocidade de fala em atividade de leitura.

\begin{abstract}
Purpose: To propose and evaluate the effectiveness of a Speech Pathology Program for Training of Radio Presenters, which was used with students from a professional radio broadcasting course. Methods: Seventy adult participants were divided into two groups, matched by age and gender: Group I (research group), composed by 35 subjects from a radio broadcasting course; Group II (control group), composed by 35 adults who spontaneously volunteered to participate on the study. The program was structured in seven modules with the duration of seven weekly lectures. The program was based on the stimulation of 11 aspects, which were presented sequentially. Three types of analysis were carried out: objective analysis of the voice, through the extraction of the fundamental frequency; perceptive-auditory analyses of the vocal use; and objective analysis of the speech rate during a reading task. Results: The results indicated that the groups differed from each other regarding vocal use and speech rate during reading. Regarding the analysis of vocal use, the groups were different on the following parameters: vocal quality, loudness, resonance, coordination between breathing and speaking, articulation, modulation and reading rhythm. Regarding the analysis of speech rate, the groups were different on the numbers of words and syllables per minute. As for the objective analysis of voice, the research group presented differences in their pre and post program application performances, with reduction of the fundamental frequency. Conclusion: The results indicate that the program was effective in its proposition, and the parameters in which improvements were observed were those specific for a good broadcasting.
\end{abstract}

Keywords: Voice training; Voice; Radio; Speak; Acoustic of speech; Speech perception; Methods; Program development

\title{
REFERÊNCIAS
}

1. Timmermans B, De Bodt M, Wuyts F, Van de Heyning P. Vocal hygiene in radio students and in radio professionals. Logoped Phoniatr Vocol. 2003;28(3):127-32.

2. Williams W, Presbury J. Observations of noise exposure through the use of headphones by radio announcers. Noise Health. 2003;5(19):69-73.

3. McHenry MA, Carlson HK. The vocal health of auctioneers. Logoped Phoniatr Vocol. 2004;29(1):41-7.

4. Kyrillos LC, Lourenço IC, Ferreira LM, Toledo FB. Posturas comunicativas de radialista de AM e FM. Pró-Fono. 1995; 7(edição especial): 28-31.

5. Oliveira IB. A Atuação fonoaudiológica com locutores de rádio. In:
Lopes Filho,O. Tratado de fonoaudiologia. São Paulo: Roca; 1997. p.751-61.

6. Navarro CA, Behlau M. O perfil vocal dos locutores: profissionais da voz em publicidade. In: Behlau M. A voz do especialista. Rio de Janeiro: Revinter; 2001. p.57-76.

7. Leite AP, Viola IC. A qualidade vocal em locutores de rádio AM e FM. Pró-Fono. 1995; 7(edição especial): 37-40.

8. Lehto L, Alku P, Bäckström T, Vilkman E. Voice symptoms of call-centre customer service advisers experienced during a work-day and effects of a short vocal training course. Logoped Phoniatr Vocol. 2005;30(1):1427. 
9. Roy N, Merrill RM, Thibeault S, Gray SD, Smith EM. Voice disorders in teachers and the general population: effects on work performance, attendance, and future career choices. J Speech Lang Hear Res. 2004;47(3):542-51.

10. Borrego, MCM. Efeitos do treinamento de voz e fala em alunos do curso de radialista [monografia]. São Paulo: Centro de Estudos da Voz; 2004.

11. Timmermans B, De Bodt MS, Wuyts FL, Boudewijns A, Clement G, Peeters A, et al. Poor voice quality in future elite vocal performers and professional voice users. J Voice. 2002;16(3):372-82.

12. Vilkman E. Occupational safety and health aspects of voice and speech professions. Folia Phoniatr Logop. 2004;56(4):220-53.

13. Jones K, Sigmon J, Hock L, Nelson E, Sullivan M, Ogren F. Prevalence and risk factors for voice problems among telemarketers. Arch Otolaryngol Head Neck Surg. 2002;128(5):571-7.

14. Medrado RB. Locução publicitária: análise perceptivo-auditiva e acústica de recursos vocais [dissertação]. São Paulo: Pontifícia Universidade Católica de São Paulo; 2002.

15. Torres ML, Behlao M, Oliveira CA. Estudo da intenção comunicativa do repórter de TV na transmissão de textos noticiosos com dois conteúdos diferentes. Fono Atual. 2004; 7(27):65-77.

16. Gustafson K. The prosody of Norwegian news broadcasts. In: Engstrand O, Kylander C, Dufberg M, editors. Papers from the Fifth National Phonetics Conference; 1991 May 29-31; Stockholm, Sweden. Stockholm: University of Stockholm; 1991. p. 49-52.

17. Kyrillos LC, Cotes C, Feijó D. Voz e corpo na TV. São Paulo: Globo; 2003.

18. DeJonckere PH, Crevier-Buchman L, Marie JP, Moerman M, Remacle M, Woisard V, et al. Implementation of the European Laryngological Society (ELS) basic protocol for assessing voice treatment effect. Rev Laryngol Otol Rhinol (Bord). 2003;124(5):279-83.
19. Roy N, Merrill RM, Gray SD, Smith EM. Voice disorders in the general population: prevalence, risk factors, and occupational impact. Laryngoscope. 2005;115(11):1988-95.

20. Williams NR. Occupational voice disorders due to workplace exposure to irritants - a review of the literature. Occup Med (Lond). 2002;52(2):99-101.

21. Telec B, Piestrzy ska H, Gajewski T. [Efficacy of the health promotion program "Prevention of Voice Disorder in Teachers and Students" implemented by the WOMP from Zielona Gora]. Med Pr. 2003;54(1):958. Polish.

22. Duffy OM, Hazlett DE. The impact of preventive voice care programs for training teachers: a longitudinal study. J Voice. 2004;18(1):63-70.

23. Sundberg J. The science of the singing voice. Dekolb: Northern Illinois University; 1987. p.146-56.

24. Welham NV, Maclagan MA. Vocal fatigue: current knowledge and future directions. J Voice. 2003;17(1):21-30.

25. Begliarov ME. [The method of voice recovery in functional dysphonias in voice professionals] Vestn Otorinolaringol. 2005;(5):50-1. Russian.

26. Timmermans B, De Bodt MS, Wuyts FL, Van de Heyning PH. Training outcome in future professional voice users after 18 months of voice training. Folia Phoniatr Logop. 2004;56(2):120-9.

27. Lehto L, Rantala L, Vilkman E, Alku P, Bäckström T. Experiences of a short vocal training course for call-centre customer service advisors. Folia Phoniatr Logop. 2003;55(4):163-76.

28. Andrade CR. Protocolo para avaliação da fluência da fala. Pró-Fono. 2000 12(2):131-4.

29. Andrade CR. Processamento da fala. Aspectos da fluência. Pró-Fono. $2000,12(1): 69-71$.

30. Andrade C, Zackiewicz DV, Sassi FC. Seis parâmetros da fluência. Rev Soc Bras Fonoaudiol. 2000; 5(7):59-64. 
Anexo 1. Protocolo de triagem fonoaudiológica

Nome:

Data:

Articulação - Lista de imitação

\begin{tabular}{|c|c|c|c|}
\hline Selo & $\operatorname{sim}(\quad)$ & não ( & ) \\
\hline Cortina & $\operatorname{sim}(\quad)$ & não ( & ) \\
\hline Porco & $\operatorname{sim}(\quad)$ & não ( & ) \\
\hline Zero & $\operatorname{sim}(\quad)$ & não ( & ) \\
\hline Nariz & $\operatorname{sim}(\quad)$ & não ( & ) \\
\hline Prego & $\operatorname{sim}(\quad)$ & não ( & ) \\
\hline Cravo & $\operatorname{sim}(\quad)$ & não ( & ) \\
\hline Travessa & $\operatorname{sim}(\quad)$ & não ( & ) \\
\hline Abelha & $\operatorname{sim}(\quad)$ & não ( & ) \\
\hline Plástico & $\operatorname{sim}($ & não ( & ) \\
\hline Bloco & $\operatorname{sim}($ & não ( & ) \\
\hline Globo & $\operatorname{sim}(\quad)$ & não ( & ) \\
\hline
\end{tabular}

Articulação - Lista de nomeação

\begin{tabular}{|c|c|c|}
\hline Tesoura & $\operatorname{sim}(\quad)$ & não ( ) \\
\hline Cadeira & $\operatorname{sim}(\quad)$ & não ( \\
\hline Vassoura & $\operatorname{sim}(\quad)$ & não ( \\
\hline Peixe & $\operatorname{sim}(\quad)$ & não ( \\
\hline Milho & $\operatorname{sim}(\quad)$ & não ( \\
\hline Garfo & $\operatorname{sim}(\quad)$ & não ( \\
\hline Blusa & $\operatorname{sim}(\quad)$ & não ( \\
\hline Tambor & $\operatorname{sim}($ & não ( \\
\hline Livro & $\operatorname{sim}($ & não ( \\
\hline Trator & $\operatorname{sim}($ & não ( \\
\hline Planta & $\operatorname{sim}($ & não ( \\
\hline Cruz & $\operatorname{sim}($ & não ( \\
\hline
\end{tabular}

Articulação - Lista de "Trava-Língua"

\begin{tabular}{|c|c|c|c|}
\hline Inclusive & $\operatorname{sim}(\quad)$ & não ( & ) \\
\hline Teatral & $\operatorname{sim}(\quad)$ & não ( & ) \\
\hline Tóxico & $\operatorname{sim}(\quad)$ & não ( & ) \\
\hline Declarações & $\operatorname{sim}(\quad)$ & não ( & ) \\
\hline Implementar & $\operatorname{sim}($ & não ( & ) \\
\hline Programa & $\operatorname{sim}(\quad)$ & não ( & ) \\
\hline Clássico & $\operatorname{sim}(\quad)$ & não ( & ) \\
\hline Privilegiadas & $\operatorname{sim}(\quad)$ & não ( & ) \\
\hline Seqüestradores & $\operatorname{sim}(\quad)$ & não ( & ) \\
\hline Fronteiras & $\operatorname{sim}($ & não ( & ) \\
\hline Problema & $\operatorname{sim}(\quad)$ & não ( & ) \\
\hline
\end{tabular}

Linguagem

\begin{tabular}{|l|ll|}
\hline Recontagem de histórias & $\operatorname{sim}(\quad)$ & não $(\quad)$ \\
\hline
\end{tabular}

Voz

\begin{tabular}{|c|c|c|}
\hline Falha & $\operatorname{sim}(\quad)$ & não ( ) \\
\hline Rouquidão & $\operatorname{sim}(\quad)$ & não ( \\
\hline Quebra de freqüência & $\operatorname{sim}(\quad)$ & não ( \\
\hline Quebra de sonoridade & $\operatorname{sim}(\quad)$ & não ( \\
\hline
\end{tabular}

Fluência

\begin{tabular}{|l|ll|}
\hline Rupturas gagas & $\operatorname{sim}(\quad$ não $(\quad)$ \\
\hline
\end{tabular}

Variações fonoaudiológicas

\begin{tabular}{|l|ll|}
\hline Uso de prótese auditiva & $\operatorname{sim}(\quad)$ & não $(\quad)$ \\
\hline $\begin{array}{l}\text { Uso de prótese dentária } \\
\text { Removível }\end{array}$ & $\operatorname{sim}(\quad)$ & não $(\quad)$ \\
\hline
\end{tabular}

Sistema miofuncional - Lábios

\begin{tabular}{|c|c|c|}
\hline Tonicidade & $\operatorname{sim}(\quad)$ & não ( ) \\
\hline Mobilidade & $\operatorname{sim}($ & não ( \\
\hline Postura & $\operatorname{sim}($ & não ( \\
\hline
\end{tabular}

Sistema miofuncional - Língua

\begin{tabular}{|c|c|c|}
\hline Tonicidade & $\operatorname{sim}(\quad)$ & não ( ) \\
\hline Mobilidade & $\operatorname{sim}($ & não ( \\
\hline Postura & $\operatorname{sim}($ & não ( \\
\hline
\end{tabular}

Sistema Miofuncional - Palato

\begin{tabular}{|l|ll|}
\hline Tonicidade & $\operatorname{sim}(\quad)$ & não $(\quad)$ \\
\hline Mobilidade & $\operatorname{sim}(\quad)$ & não $(\quad)$ \\
\hline
\end{tabular}

Audição - Reconhecimento

\begin{tabular}{|c|c|c|c|c|}
\hline Faca & $\operatorname{sim}($ & ) & não ( & ) \\
\hline Vaso & $\operatorname{sim}($ & ) & não ( & ) \\
\hline Chapéu & $\operatorname{sim}($ & ) & não ( & ) \\
\hline Jardim & $\operatorname{sim}($ & ) & não ( & ) \\
\hline Cama & $\operatorname{sim}($ & ) & não ( & ) \\
\hline Ganhar & $\operatorname{sim}($ & ) & não ( & ) \\
\hline Sofá & $\operatorname{sim}($ & ) & não ( & ) \\
\hline Zíper & $\operatorname{sim}($ & ) & não ( & ) \\
\hline Pêra & $\operatorname{sim}($ & ) & não ( & ) \\
\hline Beijo & $\operatorname{sim}($ & ) & não ( & ) \\
\hline Telha & $\operatorname{sim}($ & ) & $\overline{\text { não ( }}$ & ) \\
\hline Dormir & $\operatorname{sim}($ & ) & não ( & ) \\
\hline Linha & $\operatorname{sim}($ & ) & não ( & ) \\
\hline Nenhum & $\operatorname{sim}($ & ) & não ( & ) \\
\hline
\end{tabular}


Audição - Discriminação

\begin{tabular}{|c|c|c|c|c|}
\hline Zelo / gelo & $\operatorname{sim}($ & ) & não ( & ) \\
\hline Mata / mata & $\operatorname{sim}($ & ) & não ( & ) \\
\hline Zinco / cinco & $\operatorname{sim}($ & ) & não ( & ) \\
\hline Pano / mano & $\operatorname{sim}($ & ) & não ( & ) \\
\hline Pente / dente & $\operatorname{sim}($ & ) & não ( & ) \\
\hline Bomba / pomba & $\operatorname{sim}($ & ) & não ( & ) \\
\hline Praga / traga & $\operatorname{sim}($ & ) & não ( & ) \\
\hline Seio / cheio & $\operatorname{sim}($ & ) & não ( & ) \\
\hline Pato / bato & $\operatorname{sim}($ & ) & não ( & ) \\
\hline Ponta / ponta & $\operatorname{sim}($ & ) & não ( & ) \\
\hline Queijo / queixo & $\operatorname{sim}($ & ) & não ( & ) \\
\hline Tito / dito & $\operatorname{sim}($ & ) & não ( & ) \\
\hline Tela / tela & $\operatorname{sim}($ & ) & não ( & ) \\
\hline Faca / vaca & $\operatorname{sim}($ & ) & não ( & ) \\
\hline Pinta / tinta & $\operatorname{sim}($ & ) & não ( & ) \\
\hline Tomada / pomada & $\operatorname{sim}($ & ) & não ( & ) \\
\hline Chato / jato & $\operatorname{sim}($ & ) & não ( & ) \\
\hline Feto / teto & $\operatorname{sim}($ & ) & não ( & ) \\
\hline Dela / dela & $\operatorname{sim}($ & ) & não ( & ) \\
\hline
\end{tabular}

Anexo 2. Protocolo de avaliação objetiva da voz

Nome:

Data:
( ) Pré-teste
( ) Pós-teste

Valor da Freqüência Fundamental

Freqüência Fundamental $(\mathrm{Hz})$
Anexo 3. Protocolo de avaliação do uso vocal

Nome:

Data:
( ) Pré-teste
( ) Pós-teste

Qualidade vocal

\begin{tabular}{|c|c|c|}
\hline Adequada & $\operatorname{Sim}(\quad)$ & Não ( \\
\hline Soprosa & $\operatorname{Sim}($ & Não ( \\
\hline Rouca & $\operatorname{Sim}($ & Não ( \\
\hline Abafada & $\operatorname{Sim}($ & Não ( \\
\hline Hipernasal & Sim ( & Não ( \\
\hline Hiponasal & Sim ( & Não ( \\
\hline
\end{tabular}

Ataque vocal

\begin{tabular}{|l|ll|}
\hline Normal & $\operatorname{Sim}(\quad)$ & Não $(\quad)$ \\
\hline Brusco & $\operatorname{Sim}(\quad)$ & Não $(\quad)$ \\
\hline Soproso & $\operatorname{Sim}(\quad)$ & Não $(\quad)$ \\
\hline
\end{tabular}

Pitch vocal

\begin{tabular}{|l|ll|}
\hline Adequado & $\operatorname{Sim}(\quad$ Não $(\quad)$ \\
\hline Excessivamente Grave & $\operatorname{Sim}(\quad)$ & Não $(\quad)$ \\
\hline Excessivamente Agudo & $\operatorname{Sim}(\quad)$ & Não ( $)$ \\
\hline
\end{tabular}

Loudness vocal

\begin{tabular}{|l|ll|}
\hline Adequado & $\operatorname{Sim}(\quad)$ & Não ( ) \\
\hline Reduzido & $\operatorname{Sim}(\quad)$ & Não $(\quad)$ \\
\hline Aumentado & $\operatorname{Sim}(\quad)$ & Não $(\quad)$ \\
\hline
\end{tabular}

Ressonância vocal

\begin{tabular}{|c|c|c|}
\hline Uso equilibrado & $\operatorname{Sim}(\quad)$ & Não ( \\
\hline Foco hipernasal & $\operatorname{Sim}($ & Não ( \\
\hline Foco hiponasal & $\operatorname{Sim}($ & Não ( \\
\hline Foco nasal compensatório & $\operatorname{Sim}($ & Não ( \\
\hline Foco baixo & $\operatorname{Sim}($ & Não ( \\
\hline Foco posterior & $\operatorname{Sim}($ & Não ( \\
\hline
\end{tabular}

Coordenação pneumo-fono-articulatória

\begin{tabular}{|l|ll|}
\hline Coordenação Presente & $\operatorname{Sim}(\quad)$ & Não ( $)$ \\
\hline Incoordenação & $\operatorname{Sim}(\quad)$ & Não ( $)$ \\
\hline Uso de ar de reserva & $\operatorname{Sim}(\quad)$ & Não $(\quad)$ \\
\hline
\end{tabular}

Articulação

\begin{tabular}{|l|ll|}
\hline Precisa & $\operatorname{Sim}(\quad)$ & Não ( $)$ \\
\hline Travada & $\operatorname{Sim}(\quad)$ & Não $(\quad)$ \\
\hline Imprecisa & $\operatorname{Sim}(\quad)$ & Não $(\quad)$ \\
\hline
\end{tabular}


Modulação

\begin{tabular}{|l|ll|}
\hline Adequada & $\operatorname{Sim}(\quad)$ & Não $(\quad)$ \\
\hline Reduzida & $\operatorname{Sim}(\quad)$ & Não $(\quad)$ \\
\hline Excessiva & $\operatorname{Sim}(\quad)$ & Não $(\quad)$ \\
\hline
\end{tabular}

Ritmo de leitura

\begin{tabular}{|l|ll|}
\hline Adequado & $\operatorname{Sim}(\quad$ Não & Não ( ) \\
\hline Inadequado & $\operatorname{Sim}(\quad)$ &
\end{tabular}

Anexo 4. Protocolo para avaliação da fluência da fala

Nome:

Data:
( ) Pré-teste
( ) Pós-teste

Transcrição da amostra da fala 1 - Notícia

Cálculo da velocidade de fala

Fluxo de palavras por minuto

Fluxo de sílabas por minuto

Transcrição da amostra da fala 2 - Comercial

Cálculo da velocidade de fala

Fluxo de palavras por minuto

Fluxo de sílabas por minuto 